\title{
Assessment of Cardiovascular Disease Risk in Type 2 Diabetic Patients: A Comparison of Risk Indices
}

\author{
Brown Holy, Obi Doris Chidinma \\ Dept. of Medical Laboratory Science, Rivers State University of Science and Technology, Npkolu, Port Harcourt, Nigeria
}

Email address:

hbinternational2002@yahoo.com (B. Holy)

\section{To cite this article:}

Brown Holy, Obi Doris Chidinma. Assessment of Cardiovascular Disease Risk in Type 2 Diabetic Patients: A Comparison of Risk Indices. American Journal of Clinical and Experimental Medicine. Vol. 4, No. 6, 2016, pp. 216-220. doi: 10.11648/j.ajcem.20160406.20

Received: October 30, 2016; Accepted: November 19, 2016; Published: December 26, 2016

\begin{abstract}
Diabetes mellitus is a major public health problem; it is synchronized with the development of many complications of which cardiovascular disease is the most prevalent and detrimental. This study aimed at comparing the strength of various cardiovascular risk indices among diabetes type 2 patients. The risk indices evaluated were: Apoprotein $\mathrm{A}_{1}$, Apoprotein $\mathrm{B}_{1}$, lipoprotein (a), total cholesterol, (TCHOL), Triglyceride (TG), low density lipoprotein (HDL), TC/HDL, pentad and tetrad. Two hundred and four (204) subjects were studied comprising of 152 diabetes subjects and 52 non-diabetics as control. The result shows significantly higher values in the mean blood pressure of diabetic subjects when compared with the control at $(\mathrm{p}<0.05$. Mean HbA1C and total cholesterol values for diabetes subjects were significantly higher when compared with the control. However, the HDL-cholesterol of the diabetes subjects was significantly lower when compared to the control subjects. There was no significant difference in triglyceride level of both groups. The mean values of Apo A, Apo B and lipoprotein a, were higher in diabetic subjects compared to the control subjects at $(\mathrm{p}<0.05)$. Comparison of the liporprotein induces in terms of sensitivity, specificity and accuracy, Lpa had a sensitivity of $97.3 \%$, specificity of $100 \%$ and accuracy of $95.7 \%$, next was pentad with sensitivity of $91.45 \%$, specificity of $100 \%$ and accuracy of $95.73 \%$. Tetrad had a sensitivity of $81.05 \%$, specificity of $100 \%$ and accuracy of $90.5 \%$, the TC/HDL ratio and the least accuracy of $75.76 \%$. Based on the overall accuracy Lpa tend to display highest accuracy followed by PENTAD and TETARD.
\end{abstract}

Keywords: Diabetes, Cardiovascular Disease, Risk Indices, Lipoproteins

\section{Introduction}

Diabetes Type 2 goes on undiagnosed for many years; it is most predominant type of diabetes since it represents $90 \%$ of diabetes cases. The majority of new cases of Type 2 occur in the context of westernized lifestyles, high-fat diets and decreased exercise, leading to increasing levels of obesity, insulin resistance (IR), compensatory hyperinsulinaemia and ultimately, beta-cell failure and Type 2 [1].

The clustering of vascular risk seen in association with insulin resistance (IR), often referred to as the metabolic syndrome, has led to the view that cardiovascular risk appears early, prior to the development of Type 2, whilst the strong relationship between hyperglycaemia and microvascular disease (retinopathy, nephropathy, neuropathy) indicates that this risk is not apparent until 'frank' hyperglycaemia appears [2]. These concepts highlight the progressive nature of both Type 2 and associated cardiovascular risk, which pose specific challenges at different stages of the life of an individual with diabetes mellitus (DM). The effects of advancing age, co-morbidities and problems associated with specific groups all indicate the need to manage risk in an individualized manner, empowering the patient to take a major role in the management of his or her condition [3].

As in the world generally and Europe particularly, changes in response to demographic and cultural shifts in societies also result in the pattern of disease and their implications vary. This largely depends on the genetic variation of the various races. The Middle East, Asia-Pacific rim and parts of both North and South America have experienced massive increases in the prevalence of DM over the past 20 years, changes mirrored in European populations over the same period [4]. Awareness of specific issues associated with gender and race and, particularly, the effects of DM in women including epigenetic and influences on non- 
communicable diseases - are becoming of major importance.

Cardiovascular disease (CVD) is the leading cause of death and disability in the Western world and contributes substantially to healthcare budgets. Cardiovascular disease is defined collectively in these guidelines as coronary heart disease (CHD), stroke and other vascular disease including peripheral arterial disease (PAD) and renovascular disease, is a leading cause of death and disability in Australia and other parts of the world [5] and in 2003 accounted for approximately $18 \%$ of the total burden of disease in Australia [6].

Cardiovascular disease is the most prevalent and detrimental complication of diabetes mellitus. The incidence of cardiovascular mortality in diabetic subjects without a clinical history of previous cardiac events is as high as the incidence in non diabetic subjects with a history of myocardial infarction. This inordinate increase in the risk of coronary events in diabetic patients is attributed to multiple factors, including glycation and oxidation of proteins and increased prevalence of classic risk factors of coronary disease, such as hypertension, obesity, and dyslipidemia [7]. Despite advances in the management of cardiovascular disease, a large proportion of diabetic subjects continue to have uncontrolled hyperglycemia, hypertension, and dyslipidemia.

Lipid Tetrad Index (LTI) and Lipid Pentad Index (LPI) constitute a new and efficient evaluation of the lipid profile and cardiovascular disease risk. Lipid tetrad index is derived by the product of Total Cholesterol, Triglycerides and Lipoprotein-a divided by the High Density Lipoprotein (HDL) value. While a pentad index is determined as he product of Total Cholesterol, Triglycerides, Lipoprotein (A) and Apolipoprotein B divided by Apolipoprotein-AI. Substances such as cholesterol and triglyceride that are synthesized in the intestine or liver need to be transported to tissues and organs for their varied metabolic functions. Given the hydrophobic nature of the neutral fats, triglycerides, and cholesterol esters, lipid transport and delivery via plasma would not be possible without some form of hydrophilic adaptation. Lipoproteins are the particles that transport these lipids in the blood stream. Lipoproteins are composed of proteins (apolipoproteins or apoprotein) and lipids (phospholipids, triglycerides and cholesterol). The lipoproteins vary in the major lipoprotein present, and the relative contents of the different lipid components. Chylomicron, Low Density Lipoprotein (LDL), High density lipoprotein (HDL), Intermediate density lipoprotein, Very low density lipoprotein and free fatty acid are the classes of lipoproteins therein. Apoproteins (Apo) which attach to lipids to form lipoproteins includes; Apo A-1, Apo B-100, Apo B48, Apo C-II and Apo E.A detailed and enhanced articulation of the relationship between lipoproteins, Apoprotein, Lipids and cardiovascular disease in Type 2 diabetes Mellitus and the use of tetrad and pentad indices will clearly reveal the risk profile involved. This study focused on comparing the strength of various cardiovascular risk indices on diabetes type 2 patients.

\section{Material and Methods}

\subsection{Subjects}

The subjects were patients diagnosed of diabetes types 2 that were attending routine fasting blood sugar checks at Braithwaite Memorial Specialist Hospital, Port Harcourt.

Fasting blood sugar and HbAlc tests were done to determine the diabetic status of the patients. The subjects were addressed and the purpose of the study made known to them. Upon receipt of consents, which were freely given by the patients, they were required to complete a set of questionnaire. Weight, height, blood pressure were measured as describe below and $4-5 \mathrm{ml}$ of venous blood sample were collected and dispensed into three different sample bottles.

\subsection{Collection of Blood Specimen}

The samples were collected between the hours of 7.00am to $9.00 \mathrm{am}$. Daily samples for fasting sugars were centrifuged, the plasma was used for glucose estimations immediately while samples in the EDTA was kept in the refrigeration at 2$4^{\circ} \mathrm{C}$ for at3-4days in order to turn them in batches for glycosylated hemoglobin. The samples in plain container was allowed to clot, centrifuged, separated and stored for lipid profile, apolipoproteinA-1, Apolipoprotein B and Aplipoprotein A.

\subsection{Weight, Height and Blood Pressure}

The subjects were weighed with bathroom scale without shoes and object in their hands. They were made to stand erect when the readings were being taken with weighing balance and recorded on the questionnaire. The height was measured using a measurement tape and was recorded on the questionnaire.

The measurement of the blood pressure and was done twice at the intervals of 5minutes using automatic blood pressure monitor and the average taken.

\subsection{Determination of Biochemical Indices}

The biochemical indices determined were fasting blood glucose, fasting lipids, HbA1c, APO-A1, APO-B and lipoprotein a. All the biochemical parameters were determined using standard procedures and good laboratory practices.

\section{Results}

\subsection{Biophysical Parameters of Diabetic Subject Compared with Control}

The biophysical parameters of diabetic subjects for both control and test (diabetic) subjects are shown in table 1. The table shows that the mean \pm SD for systolic blood pressure for control and test subjects were $127.6 \pm 12.8 \mathrm{mmHg}$ and $139.5 \pm 17.8 \mathrm{mmHg}$ respectively. There was significant increase in mean systolic blood pressure of the diabetic group compared to the control at $\mathrm{p}<0.0001$. Also, mean values for 
diastolic blood pressure of the diabetics was significantly higher than the control $(\mathrm{p}=0.021)$. Comparison of the mean values of control and test subjects for both BMI and waist circumference was observed not to be statistically significant.

Table 1. Biophysical parameters of diabetic subject compared with control.

\begin{tabular}{llll}
\hline Biophysical Parameters & Diabetics N=152 & Control N=52 & p values \\
\hline Systolic Blood Pressure $(\mathrm{mmHg})$ & $139.52 \pm 17.83$ & $127.55 \pm 12.81$ & 0.0001 \\
Diastolic Blood Pressure $(\mathrm{mmHg})$ & $82.89 \pm 13.46$ & $78.13 \pm 7.63$ & $\mathrm{~S}$ \\
BMI $\left(\mathrm{kg} / \mathrm{m}^{2}\right)$ & $29.72 \pm 6.27$ & $30.27 \pm 6.01$ & 0.0211 \\
Waist Circumference $(\mathrm{cm})$ & $39.37 \pm 4.81$ & $37.42 \pm 5.61$ & $\mathrm{~S}$ \\
\hline
\end{tabular}

\subsection{Levels of Glucose and Lipid Profile of Diabetic and Control Subjects}

The levels of glucose and lipid profile of for both test and control subjects are displayed in table 2. The mean fasting blood sugar (FBS) values for control and test subjects were $4.77 \pm 0.65 \mathrm{mmol} / \mathrm{L}$ and $7.34 \pm 1.78 \mathrm{mmol} / \mathrm{L}$ respectively. The fasting blood sugar levels of the test group was significantly higher than the $(p=0.0001)$. A comparison of the total cholesterol LDL levels showed that the mean levels of the test group was significantly higher than the control $(p=0.0001)$. When compared, a significant decrease $(p=0.0001)$ was observed for HDL in the test group compared with the control. However, there was no significant difference in TG levels.
Table 2. Levels of glucose and Lipid Profile of diabetic and control subjects.

\begin{tabular}{lllll}
\hline Parameters & Diabetics N=152 & Control & P values & Remark \\
\hline FBS $(\mathrm{mmol} / \mathrm{L})$ & $7.34 \pm 1.78$ & $4.77 \pm 0.65$ & 0.0001 & $\mathrm{~S}$ \\
HbAlc $(\%)$ & $7.46 \pm 1.68$ & $5.34 \pm 0.5$ & 0.0001 & $\mathrm{~S}$ \\
Chol $(\mathrm{mmol} / \mathrm{L})$ & $5.81 \pm 0.87$ & $4.84 \pm 0.55$ & 0.0001 & $\mathrm{~S}$ \\
HDL $(\mathrm{mmol} / \mathrm{L})$ & $1.28 \pm 0.35$ & $1.34 \pm 0.29$ & 0.0001 & $\mathrm{~S}$ \\
TG $(\mathrm{mmol} / \mathrm{L})$ & $1.42 \pm 0.31$ & $1.03 \pm 0.25$ & 0.2100 & $\mathrm{NS}$ \\
LDL $(\mathrm{mmol} / \mathrm{L})$ & $3.74 \pm 0.94$ & $1.12 \pm 0.266$ & 0.0001 & $\mathrm{~S}$ \\
\hline
\end{tabular}

\subsection{Lipoprotein Indices for Diabetics and Control Subjects}

The levels of lipoprotein indices comparing test and control groups are shown in table 3. Mean Apo $\mathrm{A}_{1}$, Apo B, lipoprotein (a), Lipid tetrad indices (LTI) and lipid pentad indices (LPI)for diabetic group were significantly higher than the control $(\mathrm{p}=0.0001)$

Table 3. Lipoprotein indices for Diabetics and Control Subjects.

\begin{tabular}{|c|c|c|c|c|}
\hline Lipoprotein indices & Diabetics & Control & p-values & Remark \\
\hline Apo A-1 (g/L) & $1.59 \pm 0.46$ & $1.12 \pm 0.26$ & 0.0001 & $\mathrm{~S}$ \\
\hline Apo B (g/L) & $1.58 \pm 0.87$ & $0.7 \pm 0.16$ & 0.0001 & $\mathrm{~S}$ \\
\hline Lipo (a) (mmol/L) & $4.75 \pm 1.06$ & $1.51 \pm 0.21$ & 0.0001 & $\mathrm{~S}$ \\
\hline LTI & $33.00 \pm 18.83$ & $2.7 \pm 2.2$ & 0.0001 & $\mathrm{~S}$ \\
\hline LPI & $37.48 \pm 21.03$ & $2.71 \pm 3.16$ & 0.0001 & $\mathrm{~S}$ \\
\hline $\mathrm{TC} / \mathrm{HDL}$ & $4.69 \pm 1.86$ & $3.71 \pm 0.58$ & 0.0001 & $\mathrm{~S}$ \\
\hline
\end{tabular}

\section{Discussion}

Cardiovascular disease (CVD) is a general health problem world wild, currently escalating in low income countries as an important component of non-communicable disease burden [8]. Type II diabetes and its complications constitute a major worldwide public health problem. Patients with type II diabetes have 2 - 4 times higher risk of experiencing cardiovascular disease (CVD) than adults without diabetes $[9,10]$ and their relative risk for CVD is about twice as high [11] much of which may be preventable with appropriate treatment of dyslipidemia. The prevalence of CVD risk factors is dramatically increasing in low-and middle-income African countries, particularly in urban areas [12].

This study evaluated the cardiovascular risk profile in type 2 diabetes comparing various tools of assessment. In this study, systolic and diastolic blood pressure of diabetic subjects were significantly higher than the non-diabetic subjects $(p=0.001$ and $\mathrm{p}=0.0211$ ) respectively. The results are consonance with the findings (Sowers et al., 2001). They stated that many factors, including hypertension, contribute to this high prevalence of CVDs among diabetic subjects. Stressing further that hypertension is approximately twice as frequent in patients with diabetes compared with patients without the disease.

There was no significant difference between the mean BMI for control and that of diabetic subjects $(p=0.7678)$, but both mean BMI values indicate that the subjects were overweight. Elevated BMI is known to increase the risk of CVDs and diabetes globally. [13] showed that BMI was an excellent predictor of elevated CVD risk. Although, the measurement of waist (WC) circumference is recommended in current clinical guidelines. This study recorded a nonsignificant difference in mean WC for diabetic and nondiabetic $(p=0.5774)$ subjects.[14]reported in an observational study that progressively increasing risk of CHD, CVD and total mortality with higher $\mathrm{HbAlc}$, and no risk increase at low HbA1c levels even with longer diabetes duration, previous CVD or treatment with either insulin or OHAs. Patients achieving $\mathrm{HbAlc}<7 \%$ showed benefits for risk reduction [14]. This study recorded a significant increase in the HbA1c levels for diabetic subjects $(\mathrm{p}=0.0001)$ compared with the control. In 2012, [15] examined the association between baseline $\mathrm{HbA1c}$ level and mortality attributable to all-cause, cardiovascular disease, coronary heart disease and stroke. They concluded that high HbAlc predicted excess risk of all-cause, cardiovascular disease, coronary heart disease and stroke mortality. 
In this study, mean total cholesterol, and LDL values for test groups were significantly higher than the control $(p=0.0001)$. While the HDL level was lower in the diabetic subjects than the control $(\mathrm{p}=0.0001)$. Diabetes from abinitio has been shown to increase the risk of coronary heart disease in all populations studied. The association of low plasma levels of high-density lipoprotein (HDL) with states of impaired glucose metabolism and type 2 diabetes mellitus is well established, but the mechanistic links remain to be fully elucidated. Recent data suggests that HDL directly influences glucose metabolism through multiple mechanisms, [16]. In another related study, diabetic individuals had elevated triglycerides, LDL cholesterol and low levels of high density lipoprotein [HDL] cholesterol, [17]. The mean Apo $\mathrm{A}_{1}$, Apo $\mathrm{B}$, lipoprotein (a), lipid tetrad and lipid pentad values for diabetic were significantly higher than the control subjects $(p=0.0001)$. The need to search for these new methods of analysis has led to studies that more comprehensively describe patients' actual propensity to develop CVD, overcoming the analysis commonly based on individual lipid particles. The lipid tetrad (LTI) and pentad (LPI) indices have recently been described as a new form of assessment of lipid profiles, which have been analyzed in some populations [18, 19] A characteristic of these new indices is distinguished by the broad approach of atherogenic and non-atherogenic lipid particles, resulting in a single value. Based on the conventional lipid profile and the emerging risk factors such as Lp (a), apolipoprotein AI (apoA-1) and apoB, the LTI and LPI appear as models in global risk assessment, considering the multifactorial nature of CVD. In this study, these emerging CVD risk assessment tools appear to have stronger accuracy over the traditional risk assessment tools.

\section{Conclusion}

The results from the study indicate that the traditional method of using lipid profile to assess cardiovascular risk is not sensitive and specific, and not predictive of CVD risk especially in diabetics.

Lipid tetrad and lipid pentad proved to be more sensitive and specific predictors of risk of CVD in the study subjects, although lipid pentad was more sensitive and specific than lipid tetrad. However both indices were better predictors than the conventional lipid profile.

These findings are significant, as early detection of CVD risk is important in the treatment and management of cardiovascular diseases. Thus, modern prognostic indices such as lipid tetrad and lipid pentad are invaluable in the management of cardiovascular diseases.

\section{References}

[1] Beck-Nielsen, H., Ferrannini, E., Gabriel, R., Laakso, M., Laville, M., Mari, A., Natali, A. and Tura, A., (2010). Impaired beta cell glucose sensitivity rather than inadequate compensation for insulin resistance is the dominant defect in glucose intolerance. Diabetologia, 53, 749-756.
[2] Tapp, RJ, Dunstan, D. W., Philips, P., Tonkin, A., Zimmet, P. Z., \& Shaw, E. S (2006). Association between impaired glucose metabolism and quality of life: Results from the Australian diabetes obesity and lifestyle study. Diabetes Research and Clinical Practice (74)154-161.

[3] Bellamy, L., Casas, J. P., Hingorani, A. D. and Williams, D., (2009). Type 2 diabetes mellitus after gestational diabetes: A systematic review and meta-analysis. Lancet, 373, 1773-1779.

[4] Heianza Y, Arase Y, Fujihara K, Tsuji H, Saito K, Hsieh SD, Kodama S, Shimano H, Yamada N, Hara S (2012). Screening for pre-diabetes to predict future diabetes using various cut-off points for $\mathrm{HbAlc}$ and impaired fasting glucose: the Toranomon Hospital Health Management Center Study 4 (TOPICS 4). Diabetic Medicine (29) e279-e285.

[5] Australian Institute of Health and Welfare (2011). Cardiovascular disease: Australian facts 2011. Cardiovascular Disease Series.53.

[6] Begg, S. J., Vos, T., Barker, B., Stanley, L and Alan D (2008) Burden of disease and injury in Australia in the new millennium: measuring health loss from diseases, injuries and risk factors. Medical Journal of Australia; 188 (1), 36-40.

[7] Barter, P., Best, J., Boyden, A., Furler, J., Hossack, K. and Tonkin, A., (2005). National Heart Foundation of Australia and the Cardiac Society of Australia and New Zealand: position statement on lipid management. Heart Lung Circulation, 14(4), 275-291.

[8] Kadiri, S. (2005). Tackling cardiovascular diseases in Africa. BioMedical Journal of West Africa, 8,172-173.

[9] Fox, C. S., Coady, S, Sorlie, P. D., Levy, D., Meigs, J. B., Agostino, D., Wilson, P. W. F. \& Savage, P. J. (2004). Trends in cardiovascular complications of diabetes. Journal of American Medical Association, 292, 2495-2499.

[10] Laakso, M. (2001). Cardiovascular disease in type 2 diabetes: challenge for treatment and prevention. Journal of Internal Medicine, 249, 225-235.

[11] Liu, J, Sempos, C, Donahue, R. P, Dorn, J, Trevisan, M, \& Grundy, S. M. (2005). Joint distribution of Non-HDL and LDL cholesterol and coronary heart disease risk prediction among individuals with and without diabetes. Diabetes care, 28, 1916-1921.

[12] Ejim, E. C., Okafor, C. I., Emehel, A., Mbah, A. U., Onyia, U., Egwuonwu, T. \& Akabueze, J. (2011). Prevalence of Cardiovascular Risk Factors in the Middle-Aged and Elderly Population of a Nigerian Rural Community. Journal of Tropical Medicine, 2011, 1-5.

[13] Sowers, J. R., Murray, E., \& Edward, D. F. (2001). Diabetes, Hypertension, and Cardiovascular Disease: An Update. American Heart Association, 37, 1053-1059.

[14] Ancheta, I. B., Battie, C. A., Tuason, M. T., Borja-Hart, N. \& Ancheta, C. V. (2014). The prevalence of cardiovascular risk factors and diabetes increases with a body mass index of $>$ or $=23 \mathrm{~kg} / \mathrm{m} 2$ in Filipino American women. Ethnicity and Disease, 24(1), 48-54.

[15] Eeg-Olofsson, K., Cederholm, J., Nilsson, P. M., Zethelius, B., Svensson, A. M., Gudbjörnsdóttir, S. \&Eliasson, B. (2010). New aspects of $\mathrm{HbAlc}$ as a risk factor for cardiovascular diseases in type 2 diabetes: an observational study from the Swedish National Diabetes Register (NDR).Journal of Internal Medicine, 268, 468-470. 
[16] Xu, L., Chan, W. M., Hui, Y. F. \& Lam, T. H. (2012). Association between $\mathrm{HbAlc}$ and cardiovascular disease mortality in older Hong Kong Chinese with diabetes. Diabetes and Medicine, 29(3), 393-398.

[17] Drew, B. G., Rye, K., Duffy, A., Barter, S. J. \& Kingwell, P. B. A. (2012). The emerging role of HDL in glucose metabolism. Nature Reviews Endocrinology, 235, 1-9.
[18] Enas, E. A., Dhawan, J. \& Petkar, S. (1997). Coronary artery disease in Asian lndians: Lessons learnt and the role of lipoprotein (a). Indian Heart Journal, 49(1), 25-34.

[19] Das, B., Daga, M. K. \& Gupta, S. K. (2007). Lipid Pentad Index: a novel bioindex for evaluation of lipid risk factors for atherosclerosis in young adolescents and children of premature coronary artery disease patients in India. Clinical Biochemistry, 40(2), 18-24. 